\title{
S3-Leitlinie „Sedierung in der gastrointestinalen Endoskopie“: Was kostet die neue Leitlinie im stationären Alltag? Ein Rechenmodell und eine Analyse der Umsetzung 2011 unter den Mitgliedern der ALGK
}

\author{
S3 Guideline on "Sedation in Gastrointestinal Endoscopy": How Much Does the New Guideline Cost in Everyday \\ Hospital Work? A Calculation Model and Analysis of Implementation in 2011 among ALGK Members
}

Autoren

Institute
A. Behrens ${ }^{1}$, F. A. Kainzinger ${ }^{2}$, T. Nölling ${ }^{3}$, A. Wienke ${ }^{3}$, O. Pech' ${ }^{1}$, C. Ell ${ }^{1}$

Innere Medizin 2, Dr. Horst-Schmidt-Kliniken, Wiesbaden

Institut für Sozialmedizin, Epidemiologie und Gesundheitsökonomie, Charité - Universitätsmedizin Berlin

Fachanwalt für Medizinrecht, WIENKE \& BECKER - KÖLN, Köln

\section{Schlüsselwörter \\ - S3-Leitlinie „Sedierung \\ in der gastrointestinalen \\ Endoskopie“" \\ - Kostenanalyse \\ ๑ juristische Veränderungen}

Key words

- S3 guideline on "Sedation in Gastrointestinal Endoscopy"

- cost analysis

- legal changes

eingereicht 14.12 .2011

akzeptiert $\quad 2.7 .2012$

Bibliografie

DOI http://dx.doi.org/

10.1055/s-0032-1313080

Z Gastroenterol 2012; 50:

1002-1007 @ Georg Thieme

Verlag KG Stuttgart · New York . ISSN 0044-2771

\section{Korrespondenzadresse} Dr. Angelika Behrens Innere Medizin 2, Dr. Horst-Schmidt-Kliniken Ludwig-Erhard-Strasse 100 65199 Wiesbaden

Tel.: ++ 49/611/432420

Fax: ++49/611/432418

angelika.behrens@hsk-

wiesbaden.de

\section{Zusammenfassung}

v

Hintergrund: Die Einführung der S3-Leitlinie „Sedierung in der gastrointestinalen Endoskopie“ im Jahr 2008 hat zu wesentlichen organisatorischen, strukturellen, personellen, finanziellen und juristischen Veränderungen im Alltag von gastrointestinalen Endoskopieabteilungen geführt. Die Arbeit untersucht die ökonomische Auswirkung und die Veränderung der juristischen Sachlage. Darüber hinaus erfolgte eine Bestandsaufnahme der Umsetzung der Leitlinie durch eine elektronische Umfrage unter den Mitgliedern der Arbeitsgemeinschaft der leitenden Gastroenterologen im Krankenhaus (ALGK).

Methoden: Die finanzielle Mehrbelastung im Bereich der Personalkosten, die bei korrektem Einhalten der Sedierungsleitlinie entsteht, wurde anhand der Untersuchungszahlen aus dem Jahr 2009 einer Endoskopieabteilung eines nicht universitären Krankenhauses der Maximalversorgung berechnet. Die Darstellung der juristischen Bedeutung der Leitlinie erfolgte durch einen bez. des Medizinrechts versierten Juristen. Die Frage, inwieweit die Leitlinie im Jahr 2011 im Alltag aktiver endoskopischer Abteilungen umgesetzt wird, wurde mithilfe eines elektronisch an die Mitglieder der ALGK versendeten Evaluationsbogens ermittelt.

Ergebnisse: Die Umsetzung der S3-Sedierungsleitlinie führt zu einer erheblichen finanziellen Mehrbelastung im Bereich der Personalkosten. Bei einer Annahme von knapp 8000 stationären Endoskopien ergibt sich bei einer konservativen Rechnung ein zusätzlicher Betrag von 257462 ,- $€$ (Arbeitgeber-Bruttokosten) per anno.Die Analyse der Umfrage unter den Mitgliedern der ALGK bez. der Umsetzung der S3-Leitlinie 3 Jahre nach Publikation zeigte, dass diese in den wesentlichen Punkten, insbesondere dem Einsatz der 3. Person zur Sedierung, nicht erfolgt ist.

\section{Abstract \\ $\nabla$}

Background: The introduction of the $\mathrm{S} 3$ guideline on "Sedation in Gastrointestinal Endoscopy" in 2008 led to substantial organisational, structural, staffing-related, financial, and legal changes in the everyday work of departments of gastrointestinal endoscopy. This study examines the economic effects of this and the change in the legal situation. In addition, the extent to which the guideline has been implemented was assessed using an electronic questionnaire circulated to the members of the Working Group of Head Gastroenterologists in Hospitals (ALGK).

Methods: The increased financial burden in the area of staff costs resulting from correct compliance with the sedation guideline was calculated using research figures from an endoscopy department in a maximum-care non-university hospital, dating from 2009. Interpretation of the legal implications of the guideline was provided by lawyers familiar with medical legislation. The question of the extent to which the guideline has been implemented in everyday clinical practice in endoscopy departments in 2011 was investigated using an evaluation questionnaire sent to the members of the ALGK.

Results: Implementation of the S3 sedation guideline leads to a substantial increase in the financial burden in the area of staff costs. Assuming 8000 in-patient endoscopy procedures, a conservative estimate indicates extra costs amounting to $€ 257462$.- per year (gross costs for the employer). The analysis of the questionnaire sent to ALGK members on the implementation of the S3 guideline 3 years after its publication showed that its major points, particularly the deployment of a third staff member for sedation, have not been acted on.

Conclusions: The S3 guideline on "Sedation in Gastrointestinal Endoscopy" leads to a substantial increase in the financial burden in the area of staff 
Schlussfolgerung: Die S3-Leitlinie „Sedierung in der gastrointestinalen Endoskopie“ führt zu einer erheblichen finanziellen Mehrbelastung im Bereich der Personalkosten durch die Vorgabe einer 3. Person, die ausschließlich mit der Sedierung betraut ist. Diese Empfehlung wurde von den Autoren der Leitlinie ohne Vorliegen einer Evidenz ausgesprochen. Zudem führt sie zu einer deutlichen Veränderung der juristischen Sachlage, was im Schadensfall mit erheblichen Konsequenzen für den behandelnden Arzt verknüpft ist. Die Fragebogenevaluation unter den Mitgliedern der ALGK zeigte, dass eine Umsetzung in der stationären Gastroenterologie bisher nicht erfolgt ist. costs by requiring the presence of a third person exclusively concerned with sedation. This recommendation was issued by the authors of the guideline without any evidence being available. In addition, it leads to a clear change in the legal situation, which in case of claims arising is associated with substantial implications for the physician responsible. The questionnaire evaluation among the members of the ALGK showed that the guideline has not so far been implemented in in-patient gastroenterology.

\section{Einleitung}

\section{$\nabla$}

Endoskopien müssen heutzutage stress- und schmerzfrei für den Patienten durchgeführt werden. Dieses Ziel kann mithilfe einer periinterventionellen Sedierung erreicht werden. Darüber hinaus ermöglicht und erleichtert der Einsatz einer Sedierung die Durchführung bestimmter diagnostischer oder therapeutischer Prozeduren. Diese wesentlichen Faktoren haben dazu geführt, dass in den letzten 10 Jahren die regelhafte Durchführung einer Sedierung zum Standard im ambulanten und stationären Bereich der gastroenterologischen Endoskopie geworden ist [1].

Die Einführung der aktuellen S3-Leitlinie „Sedierung in der gastrointestinalen Endoskopie“ im November 2008 schreibt eine vollständige personelle Trennung von Sedierung und Endoskopie vor, wie sie in Analogie im chirurgischen Bereich seit Jahrzehnten besteht [2]. Der Einsatz einer zusätzlichen Fachkraft wird für die Durchführung der Sedierung unabhängig von der verwendeten Sedierungssubstanz (Opiod, Benzodiazepin, Propofol) gefordert. Zusätzlich sind in der Leitlinie Anforderungen an den Umfang der Überwachung formuliert, die über den aktuellen Standard der meisten Endoskopieeinheiten weit hinausgehen dürften. Damit entsteht eine neue, juristisch bedeutsame Sachlage, die in der Umsetzung eine erhebliche personelle, zeitliche, räumliche, apparative und damit finanzielle Mehrbelastung für jeden endoskopisch aktiven Gastroenterologen bedeutet.

Für den folgenden Artikel wurde exemplarisch an einem endoskopisch aktiven Zentrum eines nicht universitären Krankenhauses der Maximalversorgung diese finanzielle Mehrbelastung für den stationären Bereich berechnet.

Zusätzlich werden die Ergebnisse einer Umfrage unter den Mitgliedern der ALGK (Arbeitsgemeinschaft leitender gastroenterologischer Krankenhausärzte e.V.) unter dem Titel „Status Quo: Klinikalltag deutscher Krankenhäuser nach Erscheinen der S3Sedierungsleitlinie 2008“ vorgestellt. Ziel der Befragung war, den Stand der Umsetzung der S3-Leitlinie zu untersuchen.

\section{Methoden}

\section{$\nabla$}

\section{Kosten- und Erlösberechnung}

Die praktische Umsetzung der Sedierungsleitlinie führt zu Veränderungen im Bereich der Erlöse und der Kosten. Für die Betrachtung wurden die direkten Kosten bis zur Deckungsbeitragsstufe 2 (Erlöse abzüglich direkter Personal- und Sachkosten sowie Inanspruchnahme medizinischer Sekundärleistungen) untersucht. Die Auswirkungen auf Raum- und Infrastrukturkosten der Deckungsbeitragsstufe 3 sowie Veränderungen im Bereich der Allgemein- und Verwaltungskosten (Deckungsbeitrag 4) wurden in die Berechnung nicht miteinbezogen.

\section{Personalkosten}

Zusätzliche Personalkosten entstehen durch die personelle Trennung zwischen Endoskopie und Sedierung und die Nachüberwachungspflicht in der Endoskopieeinheit, die gemäß Leitlinie auch für stationäre Patienten gilt.

Grundlage zur aktuellen Berechnung der entstehenden Personalmehrkosten sind die Untersuchungszahlen der Endoskopien von stationären Patienten der gastroenterologischen Funktionsabteilung der Dr. Horst-Schmidt-Kliniken aus dem Jahr 2009. Die Daten zur Untersuchungsdauer sowie die Vor- und Nachbereitungszeiten wurden aus einer Datenbank entnommen, die 2006 im Rahmen eines bundesweiten durchgeführten Benchmark-Projekts erhoben wurden [4]. Die zusätzlich anfallende Nachüberwachungszeit im Aufwachraum wurde auf 60 min festgelegt.

Die Leitlinie fordert unabhängig vom individuellen Risiko eine dritte Fachkraft zur Durchführung einer Sedierung im Rahmen einer Endoskopie. Diese Aufgabe kann speziell geschultes Assistenzpersonal nach der Leitlinie jedenfalls dann übernehmen, wenn der Patient den ASA (American Society of Anasthesiologists)-Grad I oder II erfüllt und ein Arzt die Einleitung der Sedierung übernommen hat. Bei Patienten mit einer ASA-Klassifizierung Grad $\geq$ III soll nach der Leitlinie ein intensivmedizinisch erfahrener Arzt auch die Führung der Sedierung übernehmen. In jedem Fall darf die mit der Sedierung betraute Person keine anderen Aufgaben währenddessen wahrnehmen. Allerdings ist die Leitlinie bezüglich dieser Angaben nicht eindeutig. Nach Punkt 3.3.3.1. kann eine geschulte Pflegekraft eine Sedierung von Patienten der Kategorie ASA I-III übernehmen, während unter Punkt 1.5 die Leitlinie die pflegerische Übernahme nur für Patienten der ASA Klasse I und II erlaubt.

Die prozentuale Verteilung der ASA-Klassifikation je Untersuchungsart wurde aus einer Stichprobe einer an unserer Klinik durchgeführten Erfassung von Komplikationen im Rahmen von Sedierungen in der gastrointestinalen Endoskopie („Sedierung in der Endoskopie [Send]: eine prospektive Erfassung von Komplikationen“, bisher nicht veröffentlichte Daten) auf die Untersuchungen aus dem Jahr 2009, die die Datengrundlage dieser Berechnung darstellen, angewendet.

Die Leitlinie sieht vor, dass eine Sedierung ebenfalls von einem Arzt durchgeführt werden muss, wenn es sich um komplexe therapeutische Eingriffe handelt. Daher wurden bei allen therapeutischen Eingriffen Arztkosten für die Sedierung berechnet.

Die in der Leitlinie geforderte Nachüberwachung aller Patienten in der Endoskopieeinheit führt zu einem erhöhten Personalbedarf im Bereich der nicht ärztlichen Fachkraft. Eine Vorgabe, wie viele Patienten durch eine Fachkraft zeitgleich überwacht werden dürfen, gibt die Leitlinie nicht. Auch die verbindliche Mitteilung des BDA (Berufsverband deutscher Anästhesisten), die die Rahmenbedingungen einer postoperativen Überwachung fest- 
legt, nennt keinen definierten Personalschlüssel für die postoperative Überwachung („Der Personalbedarf richtet sich nach der Betriebszeit der Aufwacheinheit, der Anzahl der gleichzeitig zu überwachenden Patienten, deren Pflegekategorie sowie der zeitlichen Verteilung der anfallenden Überwachungszeiten.") [5]. Aus persönlichen Erfahrungen ist eine Betreuung unseres Kollektivs (im Durchschnitt 33\% der Patienten > ASA II) in der Nachüberwachung durch eine Fachkraft/6 Patienten ausreichend. Dieser Wert wurde in die Kostenberechnung einbezogen.

Für die ärztliche Tätigkeit wurde das Gehalt eines Facharztes im 4. Jahr von 5239,43€ zugrunde gelegt. Der Verdienst entspricht dem Tarifvertrag Ärzte vom 8.4.2008, dabei wurde die Lohnsteigerung zum 1.1.2009 berücksichtigt.

Die medizinischen Fachangestellten wurden nach dem Tarifvertrag der Bundesärztekammer vom 1.7.2009 berechnet. Dabei wurde eine Fachkraft im 11.-16. Berufsjahr gewählt, die in der Tätigkeitsgruppe IV („Selbständiges Ausführen von Tätigkeiten, die besonderen Anforderungen an die Handlungskompetenz und die Fach- und Führungsverantwortung stellen...") angesiedelt ist. Die Gehaltsstufe liegt bei $2140 €$.

Darüber hinaus zusätzlich anfallende Personalkosten, die durch unten genannte Faktoren begründet sind, wurden nicht miteinbezogen, da diese erheblichen Schwankungen in den einzelnen Abteilungen unterliegen. Diese weiteren Kostenfaktoren sind in ihrem Umfang in der individuellen Kostenrechnung für eine Abteilung keineswegs zu vernachlässigen.

Dazu zählen insbesondere vermehrte Aus- und Fortbildungskosten zum Thema Sedierung.

Ebenso wurde in der Berechnung nicht berücksichtigt, dass durch zum Teil gleichzeitig durchgeführte Untersuchungen personelle Engpässe entstehen, die einen weiteren Mehrbedarf nötig werden lassen. Feiertags-, Rufbereitschafts- und Nachtzuschläge, die im Rahmen von Notfallendoskopien anfallen, sind in der Kalkulation nicht abgebildet.

\section{Sachkosten}

Zusätzliche Sachaufwendungen im Bereich des medizinischen Bedarfs fallen durch die S3-Leitlinie nicht an.

\section{Geräte- und Raumkosten}

$\mathrm{Zu}$ den von der Leitlinie geforderten Überwachungsmaßnahmen gehören im apparativen Bereich Pulsoxymetriegeräte sowie EKGMonitore bei Patienten mit positiver kardialer Anamnese. Diese Geräte müssen in den Untersuchungsräumen und im Aufwachraum zusätzlich vorgehalten werden.

Die Nachüberwachung in einem gesonderten Aufwachraum erfordert zudem einen vermehrten Raumbedarf. Steht dieser nicht zur Verfügung fallen weitere Kosten durch bauliche Maßnahmen an.

\section{Erlöse}

Eine Prozedur zur Abbildung einer Sedierung mit Midazolam und/oder einem Opiat existiert nicht. Daher ergibt sich in bei diesen Sedierungssubstanzen kein Anspruch auf einen Erlös.

Das DRG-System bildet ausschließlich eine Sedierung mit Propofol unter der Prozedur 8-900 ab. Diese kann nach den Kodierrichtlinien des medizinischen Dienstes der Krankenkassen (Deutsche Kodierrichtlinie 030), die am 4.6.2009 als Folge der S3-Leitlinie überarbeitet worden sind, nur dann angegeben werden, wenn definierte Voraussetzungen erfüllt werden, die im klinischen Alltag nahezu nie realisiert werden können [3]. Das DRG-System sieht eine Erlössteigerung mit der Basis DRG G46 und G48 vor, eine Erlösvorhersage ist aber nicht möglich.
Ebenso wenig ist eine Erlösvorhersage bezüglich der Prozedur 8 - 900 bei anderen endoskopischen Eingriffen möglich.

\section{Evaluation der Leitlinienumsetzung}

An alle Mitglieder der ALGK wurde elektronisch ein Evaluationsbogen versendet, der wesentliche Kernfragen bezüglich der Umsetzung der S3-Leitlinie enthält. Abgefragt wurden Angaben zur Abteilung (Versorgungsstufe des Krankenhauses, Bettenanzahl, Stellenschlüssel), Angaben zum Umfang der endoskopischen Leistungen (Endoskopiezahlen/Jahr, prozentualer Anteil der therapeutischen Eingriffe) und Angaben zur Sedierung (Anteil der Propofolsedierungen, Anteil der mit einer dritten Person durchgeführten Untersuchungen, Fachkompetenz der Sedierungskraft, Umfang der Dokumentation der Sedierung, Art der Überwachung während und nach der Sedierung, Änderung bez. der personellen, räumlichen und apparativen Ausstattung bedingt durch die S3-Leitlinie).

\section{Ergebnisse \\ $\nabla$}

\section{Kostenberechnung}

Erlöse

Die OPS-Prozedur 8-900 kann in der klinischen Praxis nahezu nie anerkannt werden. Die niedrige Propofoldosis im Rahmen von diagnostischen Untersuchungen entspricht nicht der Anforderung der Kodierrichtlinie („Die Sedierung erfolgte in einer adäquaten Dosierung zur Einleitung und dem Erhalt einer Allgemeinanästhesie“). Selbst wenn der Eingriff eine entsprechend tiefe Sedierung notwendig macht, werden im klinischen Alltag die zusätzlichen Anforderungen („Die Sedierung wird durch einen Facharzt für Anästhesie durchgeführt. Es liegt eine Ausnahmesituation nach DKR P009 vor, welche sich durch den nicht regelhaften Einsatz der Sedierung bei der jeweiligen Untersuchungsmethode definiert.") nur in Ausnahmesituationen erfüllt sein. Ein relevanter Mehrerlös durch die Prozedur 8 - 900 ist nicht zu erwarten.

\section{Personalkosten}

2009 wurden 7765 Endoskopien bei stationären Patienten durchgeführt. Die Anwesenheit einer weiteren Fachkraft steigert den ärztlichen Personalbedarfs in Höhe von 212508 min und des nicht ärztlichen Bedarfs in Höhe von 45149 min. Dieses entspricht 2,8 Vollkräften im ärztlichen Dienst und 1,5 Vollkräften im nicht ärztlichen Bereich (inkl. Ausfallzeiten).

Die Gesamtpersonalkosten der hier betrachteten endoskopischen Funktionsabteilung erhöhen sich hierdurch um $257462 €(\mathrm{Ar}-$ beitgeber-Bruttokosten).

Bspw. ergibt sich eine durchschnittliche Kostensteigerung im Personalbereich für die Durchführung einer diagnostischen ÖGD von $31,74 €$, für die therapeutische ÖGD von $46,59 €$, für die diagnostische Koloskopie von 35,50€ und die therapeutische Koloskopie von 53,69€.

- Abb. 1 zeigt die Aufteilung der entstehenden Mehrkosten im Personalbereich auf den ärztlichen Dienst und die nicht ärztliche Fachkraft exemplarisch für die diagnostische und therapeutische ÖGD, Koloskopie und ERCP.

\section{Evaluation der Leitlinienumsetzung}

An der Fragebogenumfrage haben 136 der 210 leitenden gastroenterologischen Krankenhausärzte teilgenommen. Das entspricht etwa zwei Drittel der Mitglieder der ALGK ( $\bullet$ Tab. 1$)$. 


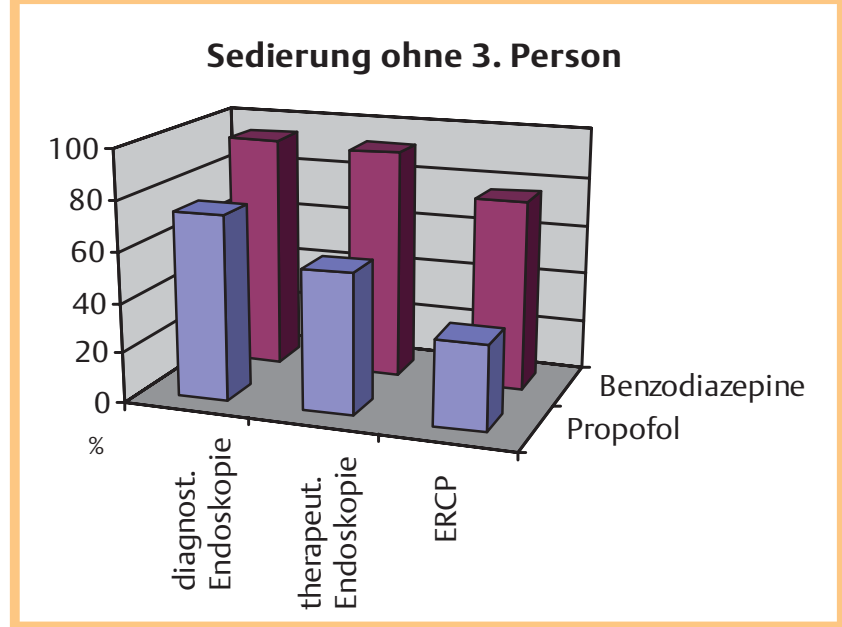

Abb. 1 Daten zur Sedierung ohne 3. Person in der diagnostischen therapeutischen Endoskopie und ERCP.

Tab. 1 Daten der Fragebogenevaluation.

\begin{tabular}{|c|c|c|}
\hline Fragestellung & $\begin{array}{l}\text { Anzahl der } \\
\text { Rückmeldungen }\end{array}$ & Ergebnis (\%) \\
\hline $\begin{array}{l}\text { Krankenhaus der Maximal-/Schwer- } \\
\text { punkt-/Regelversorgung }\end{array}$ & 136 & $16 / 46 / 38$ \\
\hline $\begin{array}{l}\text { Anzahl der Endoskopien/Jahr } \\
<1000 / 1000-5000 />5000\end{array}$ & 136 & $2 / 63 / 35$ \\
\hline $\begin{array}{l}\text { Prozentualer Anteil der therapeutischen } \\
\text { Eingriffe: }<10 / 10-30 / 30-50 />50 \%\end{array}$ & 135 & $2 / 50 / 41 / 7$ \\
\hline $\begin{array}{l}\text { Anteil der Propofolsedierungen an der } \\
\text { Gesamtzahl der Sedierungen }<30 / 30- \\
50 />50 \%\end{array}$ & 134 & $22 / 6 / 72$ \\
\hline $\begin{array}{l}\text { Sedierung mit 3. Person i. R. jeder Propo- } \\
\text { folsedierung bei diagnostischen/thera- } \\
\text { peutischen Endoskopien/ERCP }\end{array}$ & 132 & $26 / 44 / 66$ \\
\hline $\begin{array}{l}\text { Sedierung mit 3. Person i. R. jeder Mida- } \\
\text { zolam/Diazepam/Pethidinsedierung bei } \\
\text { diagnostischen/therapeutischen Endo- } \\
\text { skopien/ERCP }\end{array}$ & 75 & $6 / 8 / 24$ \\
\hline $\begin{array}{l}\text { Fachgruppenzugehörigkeit der 3. Person } \\
\text { Anästhesist/Facharzt/Assistenzarzt/ } \\
\text { Pflege }\end{array}$ & 135 & $1 / 18 / 23 / 58$ \\
\hline $\begin{array}{l}\text { Überwachung der Patienten durch Pul- } \\
\text { soxymetrie bei jeder Sedierung }\end{array}$ & 136 & 99 \\
\hline $\begin{array}{l}\text { Überwachung des Blutdrucks bei jeder } \\
\text { Sedierung }\end{array}$ & 135 & 76 \\
\hline $\begin{array}{l}\text { Überwachung mittels EKG bei kardialer } \\
\text { Anamnese bei jeder Sedierung }\end{array}$ & 132 & 42 \\
\hline $\begin{array}{l}\text { Anfertigung eines Sedierungsprotokolls } \\
\text { bei jeder Sedierung }\end{array}$ & 134 & 76 \\
\hline Dokumentation der ASA-Klasse & 136 & 45 \\
\hline $\begin{array}{l}\text { postinterventionelle Überwachung in } \\
\text { einem Aufwachraum }\end{array}$ & 136 & 54 \\
\hline $\begin{array}{l}\text { „Fitnesstest“ vor Verlegung auf eine } \\
\text { Station }\end{array}$ & 73 & 75 \\
\hline Stellenvermehrung durch die Leitlinie ja & 136 & 35 \\
\hline
\end{tabular}

Die Teilnehmer vertreten endoskopisch aktive Abteilungen, die in $63 \%$ respektive in $35 \%$ über 1000/5000 endoskopische Eingriffe pro Jahr durchführen.

$71 \%$ der Fachabteilungen setzen überwiegend (>50\%) Propofol zur Sedierung ein.
Die Kernforderung der Leitlinie ist wie dargestellt die Anwesenheit einer 3. Person zur Durchführung der Sedierung. Nach Auswertung der Fragebogen wird diese Forderung im Rahmen einer diagnostische/therapeutische Endoskopie/ERCP in 74/56/34\% im Rahmen einer Propofolsedierung nicht erfüllt. Damit wird weiterhin der überwiegende Anteil der Endoskopien in Propofolsedierung mit 2 Personen durchgeführt. Noch seltener wird diese Forderung in der Midazolam/Diazepam/Pethidin-Sedierungsgruppe umgesetzt: So findet sich eine Endoskopie ohne 3. Person in diesem Kollektiv in 94/92/76\% (diagnostische/therapeutische Endoskopie/ERCP) (○ Abb. 1).

Wird eine Sedierung durchgeführt, so wird in einem sehr hohen Prozentsatz (94\%) diese Sedierung mittels eines standardisierten Sedierungsformulars dokumentiert.

Während eine Pulsoxymetrie in annährend $100 \%$ durchgeführt wird, erfolgt eine geforderte RR-Messung in 76\% der Sedierungen gemäß Leitlinie. Dagegen findet sich nur in $42 \%$ eine Überwachung mittels EKG bei positiver kardialer Anamnese.

Postinterventionell erfolgt in 54\% der Patienten eine Überwachung in einem Aufwachraum. Von diesen Patienten wird in $74 \%$ eine Überprüfung der verbliebenen Sedierungsstärke vor Verlegung auf eine periphere Station durchgeführt.

Bezüglich einer Veränderung in Bezug auf die Personalstellen hat die Leitlinie in 35\% der Krankenhäuser zu einer Stellenvermehrung geführt; dabei sind mehrheitlich Pflegestellen (0,5 - 3 Stellen) geschaffen worden.

Im Bereich der Investitionen haben $21 \%$ der Abteilungen räumliche Veränderungen (vornehmlich die Schaffung eines Aufwachraum) realisiert. $47 \%$ der Kliniken haben neue Überwachungsgeräte angeschafft.

\section{Diskussion}

$\nabla$

Die „Deutsche Gesellschaft für Verdauungs- und Stoffwechselerkrankungen“ verpflichtet den gastroenterologisch tätigen Endoskopiker durch Implementierung der neuen S3-Leitlinie zu einem strukturierten Umgang mit dem Thema „Sedierung“. Dabei ist das Ziel, die Qualitätssicherung zu optimieren. Der Ansatz, Sedierung und Endoskopie personell zu trennen, ist insbesondere in Hinblick auf die zunehmend komplexeren endoskopischen Eingriffe grundsätzlich richtig.

In der alltäglichen Praxis führt die Umsetzung der Leitlinie aber zu einem erheblichen personellen, räumlichen und apparativen Mehraufwand, der vor dem Hintergrund der häufig wirtschaftlich angespannten Situation der Krankenhausträger die Umsetzung der juristisch bedeutsamen Leitlinie infrage stellt.

Diese aktuelle Berechnung zeigt erstmals eine Übersicht über die Art und die Höhe der anfallenden Zusatzkosten im stationären Bereich. Auch wenn diese Daten aus einer deutlich überdurchschnittlich aktiven Endoskopieabteilung stammen, so sind sie prozentual auf andere Abteilungen übertragbar.

Die Berechnung berücksichtigt ausschließlich Kosten der Deckungsbeitragsstufe 2. Kosten, die durch individuelle Gegebenheiten erheblichen Schwankungen unterliegen, wurden nicht berücksichtigt. Das sind insbesondere Kosten für die Ausund Fortbildung der Mitarbeiter, zusätzliche Feiertags-, Nachtund Rufbereitschaftskosten.

Eine Unschärfe in der Berechnung ergibt sich durch die von uns definierten Zeitrahmen der Nachüberwachung von $60 \mathrm{~min}$. Diese sollte durch prospektiv erhobene Daten untermauert werden. Daten aus einer Studie von Riphaus et al. [6] zeigen, dass $2 \mathrm{~h}$ 
nach einer Sedierung mit Propofol oder Midazolam/Pethidin im Rahmen einer Gastroskopie oder Koloskopie keine psychomotorische Beeinträchtigung des Patienten vorhanden ist. Diese Zeit dürfte sich nach Anwendung höherer Sedativadosierungen, wie bspw. bei der Push-and-pull-Endoskopie, erheblich verlängern. Die willkürliche Festlegung der Nachüberwachung auf $60 \mathrm{~min}$ unsererseits liegt somit im unteren Grenzbereich.

Zusätzlich entstehen weitere, von uns nicht berücksichtigte Mehrkosten durch die Tatsache, dass Untersuchungen gleichzeitig in mehreren Endoskopieräumen durchgeführt werden und damit der personelle Aufwand höher liegt. Dieser Faktor wurde bewusst nicht eingerechnet, um die berechneten Kosten auf andere Endoskopieabteilungen mit anderen Untersuchungszahlen übertragen zu können.

Eine Aufstellung der durch die Sedierungsleitlinie entstehenden Mehrkosten im Bereich der ambulanten Endoskopie haben Heil et al. publiziert [7]. Die Autoren berechneten u. a. einen Mehraufwand im Bereich der Personalkosten von 21,65€ je Koloskopie. Im Vergleich dazu liegen die in unserem Rechenmodell berechneten Kosten bei 35,60€. Die Kostensteigerung fällt in der zitierten Arbeit prozentual schwächer im Vergleich zu unseren Daten aus, da zum einen der überwiegende Anteil der Patienten einem ASA-Grad I oder II zuzuordnen waren und damit keine zweite ärztliche Fachkraft notwendig wird. Zum zweiten wählten die Autoren eine Nachüberwachungszeit von $20 \mathrm{~min}$ als Rechengrundlage.

Die Umsetzung der Leitlinie ist angezeigt und damit auch die anfallenden Mehrkosten. Eine S3-Leitlinie hat eine hohe Indizwirkung bez. des juristisch verbindlichen medizinischen Standards. Haftungsrechtlich darf von ihr - sofern und soweit sie den aktuellen medizinischen Standard zutreffend wiedergibt - nur in begründeten Einzelfällen abgewichen werden. Zudem liegt bereits ein juristischer Präzedenzfall (BGH, Urteil vom 8.4.2003, AZ. 6 ZR 265/02) vor, nach dem der endoskopierende Arzt für die mangelhafte Überwachung eines Patienten postinterventionell haften musste, da dieser nicht daran gehindert wurde, selber am Straßenverkehr teilzunehmen und einen Unfall verursachte, obwohl er zugesagt hatte, nicht selber zu fahren.

Die anfallenden Mehrkosten zwingen die Klinikdirektoren zur Aufnahme erneuter interner Budgetverhandlungen. Zudem müssen die Mehrkosten durch die S3-Leitlinie Eingang in die Fallkalkulation des InEK und somit in die DRG-Erlöse finden. Das DRGSystem hat grundsätzlich eine selbstlernende Systematik. Die an der Fallkalkulation beteiligten Krankenhäuser berücksichtigen die entsprechenden Mehrkosten in ihren Datensätzen, sodass diese Eingang in das Gesamtsystem finden. Hierbei ist aber mit einer Zeitverzögerung von mindestens 2 - 5 Jahren zu rechnen.

Die elektronisch durchgeführte Umfrage unter den Mitgliedern der Arbeitsgemeinschaft leitender gastroenterologischer Krankenhausärzte zeigt ein dramatisches Ergebnis. In allen wesentlichen Forderungen ist die Leitlinie mehrheitlich 3 Jahre nach ihrer Einführung nicht umgesetzt worden: Weiterhin wird der überwiegende Anteil der Endoskopien in Sedierung bei Anwesenheit von ausschließlich 2 Personen und nicht wie gefordert 3 durchgeführt. Die 3. Person ist häufig gemäß der Definition der Leitlinie nicht ausreichend qualifiziert. Postinterventionell wird nur die Hälfte der Patienten in einem Aufwachraum überwacht.

Die lebhafte Diskussion um die Leitlinie auf wissenschaftliche Veranstaltungen, die hohe Teilnehmerrate an dieser Umfrage und die aktuell bestehende gleichzeitige Existenz mehrerer bundesweiter Erhebungen zu dem Thema „Sedierung in der gastroenterologischen Endoskopie“ zeigen, dass es sich bei der fehlen- den Umsetzung nicht um eine Ignoranz der tätigen Kollegen, sondern um praktisch nicht abbildbare Forderungen durch die Leitlinie handelt. Begrenzte finanzielle Ressourcen der überwiegenden Anzahl der Krankenhäuser machen eine Umsetzung in den Alltag de facto unmöglich.

Andererseits scheint uns die zentrale Empfehlung der Leitlinie, generell bei jedweder Sedierung eine 3. Person einzusetzen, für nicht angemessen. Für diese Forderung existiert keine Evidenz: Es gibt keine Studie, die den Vorteil einer 3. Person bezüglich der Verhinderung von Komplikationen bei der Sedierung in der gastrointestinalen Endoskopie gegenüber einer „2-Personen-Prozedur“ belegt. Gerade die vielen prospektiven Studien, die in den letzten 30 Jahren zu dem Thema „Sedierung in der gastrointestinalen Endoskopie“ publiziert wurden, zeigen die hohe Sicherheit der 2-PersonenTechnik und verleihen ihr eine hohe Evidenzstärke. Das gilt insbesondere für Patienten der ASA-Klasse I-III, die einfachen diagnostischen Endoskopien unterzogen werden $[8,9]$. Diese Konstellation repräsentiert über $80 \%$ aller Endoskopien in den Facharztpraxen und ebenfalls einen ganz erheblichen Teil der Endoskopie im Klinikalltag. Eine differenzierte Empfehlung dahingehend, dass bei risikoarmen Eingriffen auf eine dritte Person verzichtet werden kann, würde ohne nennenswerte Risikoerhöhung eine erhebliche finanzielle Entlastung der Krankenhäuser und der Praxen und damit auch des Gesundheitssystems bedeuten.

Hinzu kommt die juristische Problematik bei Nichteinhaltung der Leitlinie und Auftreten einer Sedierungskomplikation. Sofern und soweit die Leitlinie den im Zeitpunkt der Behandlung aktuellen medizinischen Standard wiedergegeben hat, würde ein Verstoß gegen diese Leitlinie - sofern kein individueller Sonderfall ein Abweichen von dieser rechtfertigt - unter Umständen ein Vergehen im Sinne einer fahrlässigen Körperverletzung durch den behandelnden Arzt bedeuten. Ein Sachverhalt, der mit einer Freiheitsstrafe von bis zu 3 Jahren geahndet werden kann.

Manch ein Chefarzt fühlt sich in Sicherheit, wenn er den Geschäftsführer über die Leitlinie und die notwendige Nachbetreuung informiert. Weit gefehlt: Wenn er dennoch wie gehabt ohne Umsetzung der geforderten Änderungen weitermacht, muss er sich im Schadensfall unter Umständen als behandelnder Arzt wegen vorsätzlicher Körperverletzung verantworten, da er dann nachweisbar im Wissen um die Unterschreitung des medizinischen Standards gehandelt hat. In dieser Situation droht ein erhöhtes Strafmaß.

Zusammenfassend führt die S3-Leitlinie zur Sedierung in der gastrointestinalen Endoskopie wie dargestellt zu einer erheblichen finanziellen Mehrbelastung, die insbesondere durch die notwendige Personalaufstockung hervorgerufen wird. Dieses dürfte auch ein ganz wesentlicher Grund dafür sein, dass die Leitlinie 3 Jahre nach Einführung mehrheitlich nur in den nicht kostenintensiven Punkten umgesetzt worden ist. Dabei sollte nochmals betont werden, dass die Empfehlung zum Einsatz einer Kraft, die ausschließlich für die Sedierung verantwortlich sein soll, nicht evidenzbasiert ist. Dennoch hat eine Empfehlung einer Leitlinie juristisch einen erheblichen Wirkungsgrad. Ganz ohne Zweifel sind wir als Ärzte aufgefordert, unabhängig von einer wirtschaftlich angespannten Situation die Patientensicherheit als höchstes Gut zu verteidigen. Vor diesem Hintergrund würden die Autoren sich im Rahmen der Revision der Leitlinie einen differenzierten Umgang mit den personellen Anforderungen für die Durchführung einer Sedierung wünschen: Bei ASA-I-III-Patienten, die einen diagnostischen Eingriff oder einen nicht komplexen therapeutischen Eingriff erhalten, sollten weiterhin ein Arzt und eine Pflegekraft die Sedierung ohne eine zusätzliche mit der 
Sedierung beauftragte Person durchführen können. Prospektive Datenerhebungen sollten durchgeführt werden, um eine Ausweitung der Evidenzlage zur Bestätigung der Patientensicherheit unter den oben genannten Bedingungen zu schaffen. Unbestritten ist aus unserer Sicht, dass bei komplexen therapeutischen Eingriffen oder/und Risikopatienten der ASA-Klasse IV/V eine ausschließlich mit der Sedierung beauftragte Person anwesend ist.

\section{Danksagung}

Die Autoren bedanken sich ausdrücklich bei Herrn Dr.U. Rosien für die Bereitstellung der Daten zu den Untersuchungszeiten der endoskopischen Untersuchungen, die im Rahmen des Benchmark-Projekts 2006 evaluiert worden sind.

\section{Literatur}

1 Riphaus A, Rabofski M, Wehrmann T. Sedierung in der gastrointestinalen Endoskopie in Deutschland. Z Gastroenterol 2010; 48 (3): 392 - 397

2 Riphaus A, Wehrmann T, Weber B et al. S3-Leitlinie Sedierung in der gastrointestinalen Endoskopie 2008 (AWMF-Register-Nr. 021/014). Z Gastroenterol 2008; 46: 1298 - 1330

3 http://www.g-drg.de/cms/index.php/inek_site_de/G-DRG-System_2010/Kodierrichtlinie/Deutsche_Kodierrichtlinie_2010

4 Rosien U, Leffmann C. Benchmarking in gastrointestinal endoscopy. Z Gastroenterol 2007; 45 (12): 1228-1234

5 BDAktuell/DGAInfo. „Empfehlungen zur Überwachung nach Anästhesieverfahren.“. Anästh Intensivmed 2009; 50: 485-488

6 Riphaus A, Gstettenbauer T, Frenz MB et al. Quality of psychomotor recovery after propofol sedation for routine endoscopy: a randomized and controlled study. Endoscopy 2006; 38: 677-683

7 Heil FJ, Loibl R. Mehrkosten bei Umsetzung der S3-Leitlinie „Sedierung in der gastrointestinalen Endoskopie“. BVGD Info 2009; 2: 44-46

8 Külling $D$, Orlandi $M$, Inauen $W$. Propofol sedation during endoscopic procedures: how much staff and monitoring are necessary? Gastrointest Endosc 2007; 66 (3): 443-449

9 Rex DK, Deenadayalu VP, Eid E et al. Endoscopist-directed administration of propofol: a worldwide safety experience. Gastroenterology 2009; 137 (4): 1229-1237 